\title{
The Green Bank North Celestial Cap Pulsar Survey: Status and Future
}

\author{
Ryan S. Lynch ${ }^{1,2}$ \\ On behalf of the GBNCC Survey Collaboration \\ ${ }^{1}$ Green Bank Observatory, \\ PO Box 2, Green Bank, WV, 24944, USA \\ email: rlynch@nrao.edu \\ ${ }^{2}$ Center for Gravitational Waves and Cosmology, Department of Physics and Astronomy, West \\ Virginia University, \\ White Hall, Box 6315, Morgantown, WV 26506, USA
}

\begin{abstract}
The ongoing Green Bank North Celestial Cap pulsar survey is using the Green Bank Telescope to search for pulsars and transients over $85 \%$ of the celestial sphere. The survey has resulted in over 150 new pulsars, among which are high-precision millisecond pulsars, several binary pulsars, including at least one relativistic double neutron star system, nulling pulsars, and several nearby millisecond pulsars. We find no fast radio bursts in the survey to date. We present these results and discuss the future prospects for the survey.
\end{abstract}

\section{Introduction}

Large-area surveys are one of the best ways of discovering new pulsars. Pulsar surveys are sensitivity limited, and only $\sim 5 \%$ of the total Galactic pulsar population has been discovered, so new surveys often uncover unique and scientifically valuable systems (Lyne et al. 2004; Archibald et al. 2009; Ransom et al. 2014). Rotating radio transients (RRATs) and fast radio bursts (FRBs) were also first discovered in large-area pulsar surveys (McLaughlin et al. 2006; Lorimer et al. 2007; Thornton et al. 2013), and such surveys remain important for discovering additional transients.

The Green Bank North Celestial Cap (GBNCC) pulsar survey uses the Robert C. Byrd Green Bank Telescope ${ }^{\dagger}$ (GBT) in the 300-400 MHz band (Stovall et al. 2014). The survey began in 2009, covering declinations $\delta>38^{\circ}$, and has since expanded to cover the full sky visible to the $\operatorname{GBT}\left(\delta>-46^{\circ}\right.$, or $85 \%$ of the celestial sphere). As a low-frequency survey, the GBNCC survey preferentially detects pulsars with steep radio spectra and relatively low dispersion measure (DM). To-date, the survey has uncovered 156 new pulsars, of which 20 are millisecond pulsars (MSPs, $P<10 \mathrm{~ms}$ ), and 11 are RRATs ${ }^{\ddagger}$. We present an update on the progress of the survey and highlight some interesting results.

\section{Survey Progress and Recent Results}

Approximately 93,000 individual pointings (nearly 3100 hours) have been observed to date, making the survey $75 \%$ complete. Data are processed using a PRESTO-based $₫$ pipeline, and data are searched to a maximum DM of $3000 \mathrm{pc} \mathrm{cm}^{-3}$ (a portion of the

${ }^{\dagger}$ The Green Bank Observatory is a facility of the National Science Foundation operated under cooperative agreement by Associated Universities, Inc.

${ }^{\ddagger}$ An updated list can be found at http://astro.phys.wvu.edu/GBNCC/

Ihttp://www.cv.nrao.edu/ sransom/presto/ 
Table 1. High-Precision MSPs Discovered in the GBNCC Survey

\begin{tabular}{|c|c|c|c|c|}
\hline PSR & $\mid P(\mathbf{m s})$ & | DM & $\left(\mathrm{pc} \mathrm{cm}{ }^{-3}\right)$ & PTA Status \\
\hline $\mathrm{J} 0645+5158$ & 8.85 & | & 18.2 & | Included in NANOGrav/IPTA \\
\hline $\mathrm{J} 0740+6620^{1}$ & 2.88 & & 15.0 & | Included in NANOGrav/IPTA \\
\hline $\mathrm{J} 1125+7819^{1}$ & 4.20 & & 11.2 & | Included in NANOGrav/IPTA \\
\hline J0636+5128 & 2.87 & | & 11.1 & | Included in NANOGrav/IPTA | \\
\hline J0406+3039 & 2.61 & | & 49.4 & | Released to NANOGrav/IPTA \\
\hline J0125-2327 & 3.68 & | & 9.6 & Undergoing evaluation \\
\hline J1221-06 & 1.93 & | & 16.4 & Undergoing evaluation \\
\hline $\mathrm{J} 2022+25$ & 2.65 & | & 53.7 & Undergoing evaluation \\
\hline J1317-02 & 2.91 & I & 29.4 & Undergoing evaluation \\
\hline J2150-03 & 3.51 & | & 20.7 & Undergoing evaluation \\
\hline
\end{tabular}

Notes:

${ }^{1}$ Updated timing solutions for these pulsars will appear in Lynch et al. (in prep.)

earliest data were searched to a maximum DM of $500 \mathrm{pc} \mathrm{cm}^{-3}$ ) for both periodic and transient emission. Details of the survey strategy and pipeline can be found in Stovall et al. (2014). Pulsar timing observations have been carried out primarily with the GBT and with the Low Frequency Arrayll (LOFAR). Figure 1 shows a $P-\dot{P}$ diagram for a subset of the pulsars discovered so far. We discuss some specific results below.

\subsection{High-Precision MSPs}

The primary science goal of the GBNCC survey is the discovery of high-precision MSPs that are suitable for inclusion in pulsar timing arrays (PTAs) that are being used to directly detect low-frequency gravitational waves (GWs). MSPs that exhibit individual pulse time of arrival uncertainties $\lesssim 1 \mu$ s at $1500 \mathrm{MHz}$ are released to the North American Nanohertz Observatory for Gravitational Waves (NANOGrav; McLaughlin 2013) and the International Pulsar Timing Array (IPTA; Manchester \& IPTA 2013) after an initial period of evaluation. Four such MSPs are currently included in NANOGrav/IPTA timing, and one more has been released to the PTA collaborations (see Table 1). An additional six MSPs are currently undergoing internal evaluation and appear to be promising candidates for PTA inclusion. The success of the GBNCC survey in finding new PTA-quality MSPs is especially important, since several of these pulsars are found at $\delta>45^{\circ}$, where there is a relative paucity of PTA sources when compared to southern declinations. Full sky coverage is important for characterizing the quadripolar signature that is expected for GWs (Hellings \& Downs 1983).

\subsection{Binary Pulsars}

Twenty-two binary pulsars have been discovered to-date. These include several MSPwhite dwarf (WD) binaries, two black widow systems, and three partially recycled pulsars. One such pulsar is PSR J0509+3801, a member of a new double neutron star system (DNS). The orbital period is $P_{\mathrm{B}}=9.11 \mathrm{hr}$ and the eccentricity is $e=0.586$. This has allowed us to measure two post-Keplerian parameters: the change in the longitude of

"LOFAR (van Haarlem et al. 2013) is designed and constructed by ASTRON. It has facilities in several countries, that are owned by various parties (each with their own funding sources), and that are collectively operated by the International LOFAR Telescope foundation under a joint scientific policy. 

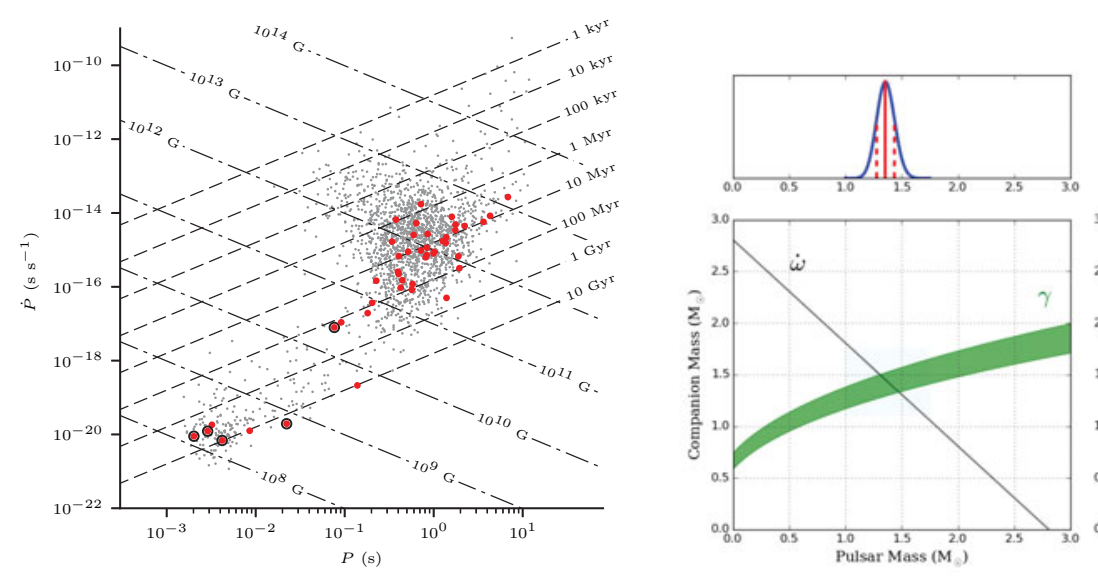

J0509+3801
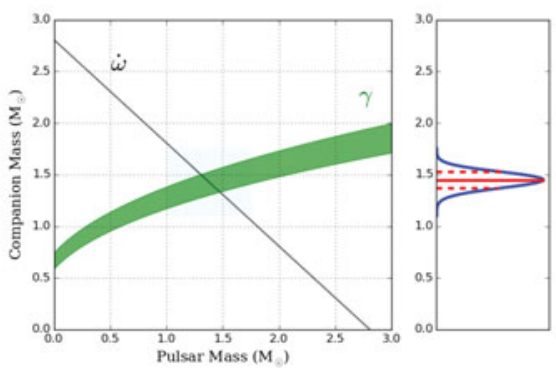

Figure 1. Left: $P-\dot{P}$ diagram showing a subset of GBNCC discoveries appearing in Lynch et al. (in prep.) in red (large points). Circles indicate binary systems. Additional pulsars from the ATNF catalog are shown in gray (small points). Right: Estimates of the posterior probability density and marginalized distribution functions (blue-sold curves) for the masses of PSR J0509+3801 and its companion. Allowed mass regions are shaded, and the thickness of each curve represents the 1- $\sigma$ uncertainty from TEMPO. The median and $68.3 \%$ credible intervals are also shown in the top panels as solid and dashed lines, respectively.

periastron $(\dot{\omega})$, and the Einstein delay due to gravitational redshift and time dilation $(\gamma)$. The combination of these two parameters provides a precise mass measurement of both stars, and we find $M_{\mathrm{p}}=1.34(8) \mathrm{M}_{\odot}$ and $M_{\mathrm{c}}=1.46(8) \mathrm{M}_{\odot}$ (see Figure 1 ). We have completed a Long Baseline Observatory campaign to measure the proper motion and parallax of PSR J0509+3801; analysis of the results are ongoing. Two other binary systems may have massive companions and are promising DNS candidates. One of the systems has $P_{\mathrm{B}}=8.9 \mathrm{~d}, e=0.25$, and a minimum companion mass $M_{\mathrm{c}, \mathrm{min}}=1.2 \mathrm{M}_{\odot}$, while another has $P_{\mathrm{B}}=10.3 \mathrm{~d}$, a nearly circular orbit, and $M_{\mathrm{c}, \mathrm{min}}=0.81 \mathrm{M}_{\odot}$.

Three binary systems have been detected at optical wavelengths. PSR J1816+4510 (Stovall et al. 2014) is in a black widow system with an interesting, metal rich companion star that does not readily fit into typical evolutionary scenarios (Kaplan et al. 2012, 2013). PSR J0214+5222 (Stovall et al. 2014) has a 512-day orbital period and a companion that is likely to be a He WD; a second long orbital period system $\left(P_{\mathrm{B}}=527 \mathrm{~d}\right)$ has been discovered, but no counterpart has yet been detected. PSR J1641+8049 (Lynch et al. in prep.) is a second black widow system with a faint optical counterpart. A weak Fermi counterpart is also detected in gamma-rays.

\subsection{Nulling Pulsars}

We have performed a preliminary nulling analysis of a sample of 44 pulsars with long, multi-year follow-up, which has resulted in the identification of six new nulling pulsars. Multi-frequency data at 350 and $820 \mathrm{MHz}$ allow us to compare the nulling fraction at different bands, and we find no evidence for frequency-dependent nulling fractions. We have also identified one new mode-changing pulsar, which switches between at least two modes, one in which a bright interpulse is observed, and another where the interpulse is very weak and often not detected. 


\subsection{Implications for the Spectra of FRBs at Low Frequencies}

No FRBs have been detected to-date in the GBNCC survey. Chawla et al. (2017) conducted a detailed analysis of over 2000 hours of survey data (84-days equivalent) to arrive at a $95 \%$ confidence upper limit on the FRB rate at $350 \mathrm{MHz}$ of $3.6 \times 10^{3} \mathrm{sky}^{-1} \mathrm{day}^{-1}$ above a peak fluence of $0.63 \mathrm{Jy}$. Ignoring the potential effects of scattering and free-free absorption, this result requires a spectral index $\left(S_{\nu} \propto \nu^{\alpha}\right)$ of $\alpha>+0.35$ to be consistent with the $1.4 \mathrm{GHz}$ rate reported by Crawford et al. (2016). If scattering models are taken into account, the constraint is $\alpha>-0.3$. In either case, Chawla et al. (2017) find that a relatively flat spectral index, which contrasts with the steeper spectral indices of radio pulsars, is required to explain the lack of FRBs in the GBNCC survey. Alternatively, free-free absorption could cause a turnover at low frequencies, which would imply that FRBs are preferentially found in regions with high optical depth at these frequencies. Chawla et al. (2017) use these results to predict the rate of detectable FRBs for current and upcoming telescopes. Under different models for scattering, the Canadian HI Intensity Mapping Experiment (CHIME) is predicted to detect $\sim 1-50 \mathrm{FRBs}$ per day in the 400-800 MHz band, which makes it one of the most promising upcoming telescopes for increasing the known population of FRBs.

\section{Survey Completion and Future Work}

At the current rate of progress, the GBNCC survey should be completed in 2-3 years. Long-term pulsar timing observations are ongoing for all the pulsars found to-date, and these efforts will continue as additional pulsars are found. The initial predictions for the survey yield may prove to be optimistic. Possible causes are an increase in RFI in the 360-380 MHz band or a genuine turnover in the number of detectable pulsars at low frequencies relative to $1.4 \mathrm{GHz}$, on which most population estimates are based. A detailed empirical analysis of the survey sensitivity and implications for the pulsar population will be the subject of future work. An improved single pulse analysis and a new implementation of the fast folding algorithm have recently been incorporated into the survey pipeline. A full reprocessing of the entire survey, which will include improved RFI mitigation and candidate selection, is planned for the near future. The GBNCC survey will also form a legacy data set that will continue to yield valuable science well into the future.

\section{References}

Archibald, A. M., Stairs, I. H., Ransom, S. M., et al. 2009, Science, 324, 1411

Chawla, P., Kaspi, V. M., Josephy, A., et al. 2017, ApJ, 844, 140

Crawford, F., Rane, A., Tran, L., et al. 2016, MNRAS, 460, 3370

Hellings, R. W. \& Downs, G. S. 1983, ApJ, 265, L39

Kaplan, D. L., Bhalerao, V. B., van Kerkwijk, M. H., et al. 2013, ApJ, 765, 158

Kaplan, D. L., Stovall, K., Ransom, S. M., et al. 2012, ApJ, 753, 174

Lorimer, D. R., Bailes, M., McLaughlin, M. A., Narkevic, D. J., \& Crawford, F. 2007, Science, 318,777

Lynch, R. S., et al. in prep.

Lyne, A. G., Burgay, M., Kramer, M., et al. 2004, Science, 303, 1153

Manchester, R. N., IPTA. 2013, Classical and Quantum Gravity, 30, 224010

McLaughlin, M. A. 2013, Classical and Quantum Gravity, 30, 224008

McLaughlin, M. A., Lyne, A. G., Lorimer, D. R., et al. 2006, Nature, 439, 817

Ransom, S. M., Stairs, I. H., Archibald, A. M., et al. 2014, Nature, 505, 520

Stovall, K., Lynch, R. S., Ransom, S. M., et al. 2014, ApJ, 791, 67

Thornton, D., Stappers, B., Bailes, M., et al. 2013, Science, 341, 53

van Haarlem, M. P., Wise, M. W., Gunst, A. W., et al. 2013, A\&A, 556, A2 